\title{
Review
}

\section{Cerebellar Afferents to Neuroendocrine Cells: Implications for Adaptive Responses to Simulated Weightlessness}

\author{
Toshiniko KATAFUCHI, Tetsuro HORI, YUtAKa OOMURA*, AND KiYomi KOIZUMI** \\ Department of Physiology, Kyushu University Faculty of Medicine, Fukuoka 812-82, \\ *Institute of Bio-Active Science, Nippon Zoki Pharmaceutical Co., Hyogo 673-14, Japan and \\ **Department of Physiology, State University of New York, Health Science Center at Brooklyn, Brooklyn, \\ NY 11203, USA
}

\section{Introduction}

IT IS WELL known that the hypothalamic paraventricular nucleus (PVN) is deeply involved in various homeostatic and/or stress responses. Magnocellular and parvocellular neurons in the PVN receive not only humoral signals from short and long loop feedback but also neuronal afferents from several regions in the brain; e.g., from the limbic system such as the amygdala and septum, circumventricular organs such as the subfornical organ and organum vasculosum laminae terminalis, other hypothalamic nuclei, and lower brain structures such as the dorsal raphe nucleus, locus ceruleus, medullary reticular formation and nucleus tractus solitarius [1]. After these signals are integrated, the PVN controls the secretion of pituitary hormones and the activity of the autonomic nerves by its direct and indirect projections to the median eminence, posterior pituitary and autonomic preganglionic neurons.

Weightlessness has been shown to produce a wide range of neuroendocrine and autonomic responses such as cardiovascular, body fluid and metabolic changes. These reactions are attributed to changes in inputs from baroreceptors and volume receptors in the thoracic cavity, propriore-

This article was written as a memorial article for the Kawakami Prize of the Japan Endocrine Society awarded to the first author (Dr. T. Katafuchi) on December 3, 1994. Correspondence to: Dr. Toshihiko KATAFUCHI, Department of Physiology, Kyushu University, Faculty of Medicine, Fukuoka 812-82, Japan ceptors in the muscles and tendons, and vestibular organs, which are normally required for posture control, i.e., orthostatic cardiovascular reflexes. Although the inputs from baroreceptors [2, 3], muscle afferent nerves [4] and somatosensory systems [5] to the hypothalamic nuclei, including the PVN, have been reported since the 1960s, direct connections between the hypothalamus and cerebellum were first shown anatomically in the 1980s [6]. Subsequently, the functional significance of the cerebello-hypothalamic projections including roles of the cerebellar input to the neurosecretory cells in the regulation of vasopressin release has been demonstrated by electrophysiological studies $[7,8]$.

In this review article, the importance of the cerebello-hypothalamic neuronal system during simulated weightlessness and orthostatic reflexes on earth will be demonstrated, and a possible role of this system in the regulation of the neuroendocrine and autonomic nervous systems will also be discussed.

\section{Paraventricular Neuron Activity During Body Suspension}

Body suspension as an experimental model for ground-based low-G simulation

It is clear that studying the responses to true weightlessness is virtually impossible in the presence of one-G on earth. A variety of methods for simulating weightlessness has therefore been de- 
veloped: e.g., water immersion, bed rest or chair rest, immobilization, tail suspension, and horizontal (HOR) or head-down tilt (HDT) body suspension [9]. It has thus been shown that the responses to space flight in man and animals including 1) cardiovascular deconditioning (a decrease in cardiac output and edema), 2) a decalcification of the bone, 3) muscle atrophy, 4) a negative water, sodium and potassium balance, and 5) weight loss are well reproduced by the experimental conditions mentioned above.

To examine the involvement of the PVN in these reactions, we recorded the single unit activity of PVN neurons during HOR and HDT suspension by means of microwire electrodes in unanesthetized rats [10].

\section{Neuron activity in the PVN during HDT suspension}

Rats in a denim harness with 4 leg openings were suspended horizontally and $45^{\circ}$ head-down to simulate a low-G environment. The animals' heads were fixed in a horizontal position. About $40 \%$ of the PVN neurons responded to HDT, of which $85 \%$ showed a gradual decrease in the firing rate, while $15 \%$ displayed a gradual increase. Some of the neurons which were suppressed by HDT also exhibited a phasic firing pattern of spontaneous activity (Fig. 1A).

Acute HDT induces central blood volume shifts which must stimulate atrial volume receptors or baroreceptors. The activation of baroreceptors has been shown to inhibit PVN neurosecretory cells [2, 3] and the secretion of vasopressin is suppressed by HDT in man [11]. Although our study did not show whether or not the PVN neurons, which responded to HDT, were neurosecretory, they were at least responsive to an intraperitoneal injection of hypertonic saline (Fig. 1B) [10], which thus indicated a convergence of cardiovascular and osmotic stimuli onto the same neuron. It is thus suggested that the HDT-sensitive neurons may be neurosecretory and that the decrease in activity during HDT is caused, at least in part, by information from baroreceptors and volume receptors. However, about half of the neurons showing a decrease in activity during HDT were also suppressed during HOR suspension, which was not considered to induce central fluid shifts. The decrease in activity was not affected by touching the hindlimbs on a surface without flexing the joints during suspension (Fig. 1A). Signals from sources other than baroreceptors, for example, from proprioceptors in extensor muscles, tendon organs and joints, but not from touch receptors, could therefore contribute to this response during HOR.

Furthermore, changes in the concentration of

A

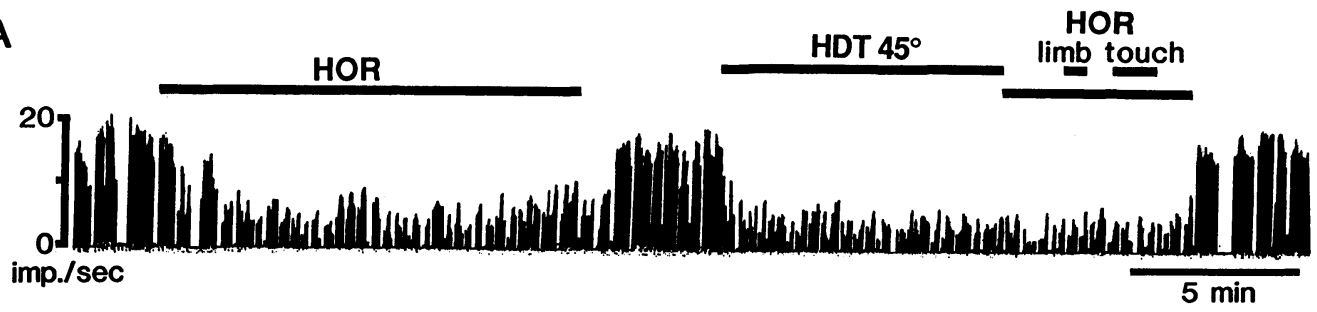

B

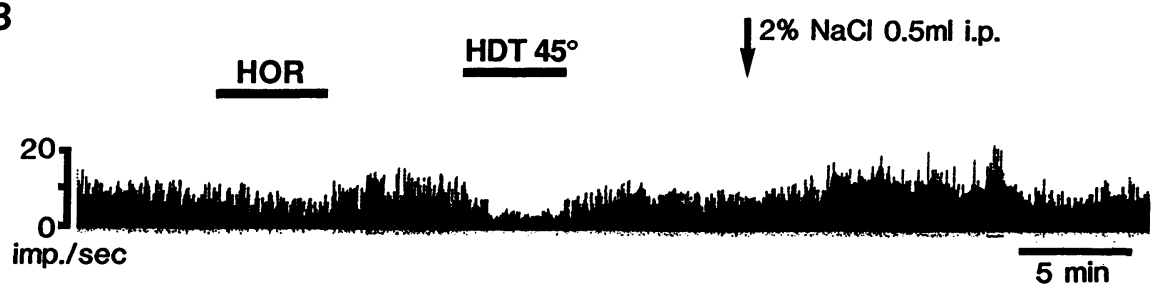

Fig. 1. The effects of body suspension and osmotic stimuli on paraventricular neuronal activity. The horizontal bars above each trace indicated horizontal suspension (HOR); HDT $45^{\circ}$, head-down tilt suspension (HDT) at $45^{\circ}$. A: phasic firing pattern with a gradual decrease in activity during HOR and HDT. A light touching of the limbs on the surface during HOR induced no change. B: a gradual decrease in the activity by HOR and a greater response by HDT. An injection of hypertonic saline (i.p.) increased activity. Modified from ref [10] with permission. 
plasma vasopressin during acute central fluid shifts in heart-lung transplant subjects were similar to those in cardiac-innervated controls [12]. Therefore, the regulation of vasopressin secretion during exposure to simulated weightlessness cannot be completely explained by input from cardiac baroreceptors. Since the neurons in the cerebellar fastigial nucleus (FN) have been shown to respond to headtilting [13], and an electrical stimulation of the FN results in changes in the plasma concentration of vasopressin [14, 15], another possibility is that the vestibular inputs from the FN may be also responsible for changing the PVN neuron activity. In fact, direct connections from the FN to the PVN neurosecretory cells have been found in an electrophysiological study [8] (see section IV).

\section{Neuronal Afferents to the PVN}

\section{Possible pathways involved in the changes in neuronal activity during body suspension}

The body suspension study revealed that neurons in the PVN [10] and the lateral hypothalamic area (LHA) [16] altered their activity in response to HDT suspension. The responses were considered to be caused by changes in the inputs from the baro- and volume receptors, muscle afferents, and vestibular organs. Furthermore, PVN neurons are sensitive to other somatosensory inputs such as pain (tail pinch) in rats [10]. It has also been reported that the neuronal activity of neurosecretory cells in the supraoptic nucleus is affected by the stimulation of polymodal fibers from skeletal muscle in cats [17].

Concerning the pathways by which these different modalities affect the PVN neurons, several possibilities exist. For example, the signals from thoracic baroreceptors which perceive blood redistribution enter the nucleus tractus solitarius, and are then sent to the PVN directly, or via a relay nucleus such as the parabrachial nucleus in the pons $[18,19]$. Input from the somatosensory system is considered to reach the hypothalamus multisynaptically via the mesencephalic reticular formation [5]. Degeneration studies have demonstrated the reticulo-hypothalamic pathways which are the so-called extralemniscal sensory system [20]. Proprioreceptive information which is proposed to alter the PVN neuron activity may also pass through this pathway. However, since the signals coming from muscle spindles are strongly associated with the vestibulo-cerebellar system, there is another possibility, i.e., these signals and the vestibular information itself are conveyed to the hypothalamus via the vestibular or the cerebellar nuclei. An electrophysiological study has shown that the lateral vestibular nucleus (Deiters') has polysynaptic connections with the LHA in rats [21]. Furthermore, it has also been suggested that baroreceptor afferents from the carotid sinus nerves reach the cerebellar cortex [22], so that at least some of the information from baroreceptors may also be sent to the hypothalamus through the cerebellum.

\section{Pathways from the cerebellum to the hypothalamus}

In addition to the well established role of the cerebellum in somatic motor regulation, it is also known that the cerebellum can modulate various autonomic responses. Stimulation of the anterior lobe of the cerebellar cortex induces changes in visceral functions such as blood pressure, respiration and intestinal motility. These responses may be caused by polysynaptic pathways from the output nuclei of the cerebellum, e.g., the FN, to the parasympathetic (dorsal motor nucleus of the vagus in brain stem) or sympathetic preganglionic neurons (intermediolateral cell column of the spinal cord) [see review ref. 6]. For example, the FN has been reported to have connections with medullary nuclei, including the lateral reticular nucleus, paramedian reticular formation, and nucleus tractus solitarius [23-25], which are closely related to the autonomic activities.

It is therefore considered that the cerebellar information concerning the autonomic component is sent not only to the brain stem but also to the upper brain structures, e.g., the hypothalamus. Since the medullary nuclei mentioned above have connections with the hypothalamic regions, such as the PVN and the lateral hypothalamic area [18, $20]$, the cerebellar signals are thought to be conveyed via these nuclei. However, several studies using horseradish peroxidase technique have demonstrated direct (monosynaptic) and reciprocal connections between the cerebellum and the hypothalamus $[6,26]$. The cerebellar nuclei including lateral, interposed and medial (FN) nuclei send their axons to the lateral hypothalamic area, dorsomedial hypothalamic nucleus, ventromedial 
hypothalamic nucleus and periventricular hypothalamic nucleus with a contralateral preponderance. On the other hand, the hypothalamic regions such as the lateral and posterior hypothalamic area, ventromedial, dorsomedial and mammillary nuclei have direct projections to the anterior and posterior lobe of the cerebellar cortex which are exclusively ipsilateral. We recently found a monosynaptic projection from the FN to the neurosecretory cells in the contralateral PVN by means of electrophysiological techniques.

\section{Fastigial Inputs to the Neurosecretory Neurons}

\section{Fastigial pressor response (FPR)}

Several lines of evidence have indicated that the $\mathrm{FN}$ of the cerebellum is involved in the central regulation of cardiovascular functions associated with somatic-postural control. Electrical stimulation of the rostromedial region of the FN (usually by train pulses at 50 to $100 \mathrm{~Hz}$ for $10 \mathrm{sec}$ ) raises the arterial blood pressure through the activation of the sympathetic nervous system, the so-called fastigial pressor response (FPR) [27], and an increase in the cerebral blood flow [28], which were similar to responses in the orthostatic reflex. In addition, the orthostatic responses were attenuated by destruction of the FN [29]. Although it was suggested that the pressor response was not due to stimulation of perikarya in the FN but to that of axons of passage in cats [30] and rats [31], the pressor response was also induced by a microinjection of an excitatory amino acid, glutamate, into the $\mathrm{FN}$ in dogs [32], and the FN neurons altered their neuronal activity in response to head-tilting [13]. It is therefore still possible that the FN takes part in postural and cardiovascular integration during the orthostatic reflex.

Circulating vasopressin, together with the sympathetic nervous system and the renin-angiotensin system, is known to play a role as an effector system of the orthostatic reflexes. Especially in quadriplegic subjects, this hormone is deeply involved in the maintenance of blood pressure in the head-up position [33]. Although stimulation of the FN has been found to modulate the secretion of vasopressin, the results have been contradictory. Hata and Miura first reported that the release of vasopressin evoked by bilateral carotid occlusion was suppressed by an electrical stimulation of the FN [14], while in another study the stimulation of the FN was found to result in an increase in plasma vasopressin levels [15].

\section{Unit activity during FPR}

Neurosecretory cells were identified by the antidromically evoked action potentials following pituitary stalk stimulation in anesthetized rats. About $40 \%$ of the spontaneously firing neurosecretory cells in the PVN, including phasic firing neurons, were suppressed during the period of train stimulation of the contralateral FN [8]. Stimulation of the $\mathrm{FN}$ with a single pulse at $1 \mathrm{~Hz}$, which did not change either the blood pressure or heart rate at all, produced an inhibitory response demonstrated by peristimulus time histograms. In addition, the suppression of the firing rates induced by train stimulation was also observed in animals denervated of the bilateral carotid sinus and vagus nerves. The inhibitory responses produced by a repetitive stimulation of the FN is therefore not secondary to hypertension. Both groups of neurosecretory neurons suppressed and unaffected by the systemic injection of phenylephrine were inhibited by FN stimulation, which suggested that not only vasopressin-secreting but also oxytocinsecreting neurons receive an inhibitory input from the FN (Fig. 2).

The inhibitory influence of the FN on the PVN neurosecretory cell as demonstrated in our study agreed with finding that the stimulation of the FN suppressed the secretion of vasopressin [14], but Del Bo et al. reported an increase in vasopressin caused by FN stimulation. They found that the residual FPR, which was followed by the rise in blood pressure during FN stimulation that caused a second peak of hypertension in about $30 \%$ of the animals tested, could be due to a rise in the plasma vasopressin levels [14]. In our study $28 \%$ of the neurosecretory neurons inhibited by a train stimulation of the FN showed a rebound excitation lasting for 1-5 min after the termination of repetitive stimulation. Although its mechanism is not known, it is possible that this rebound phenomenon is related to an increase in vasopressin. 

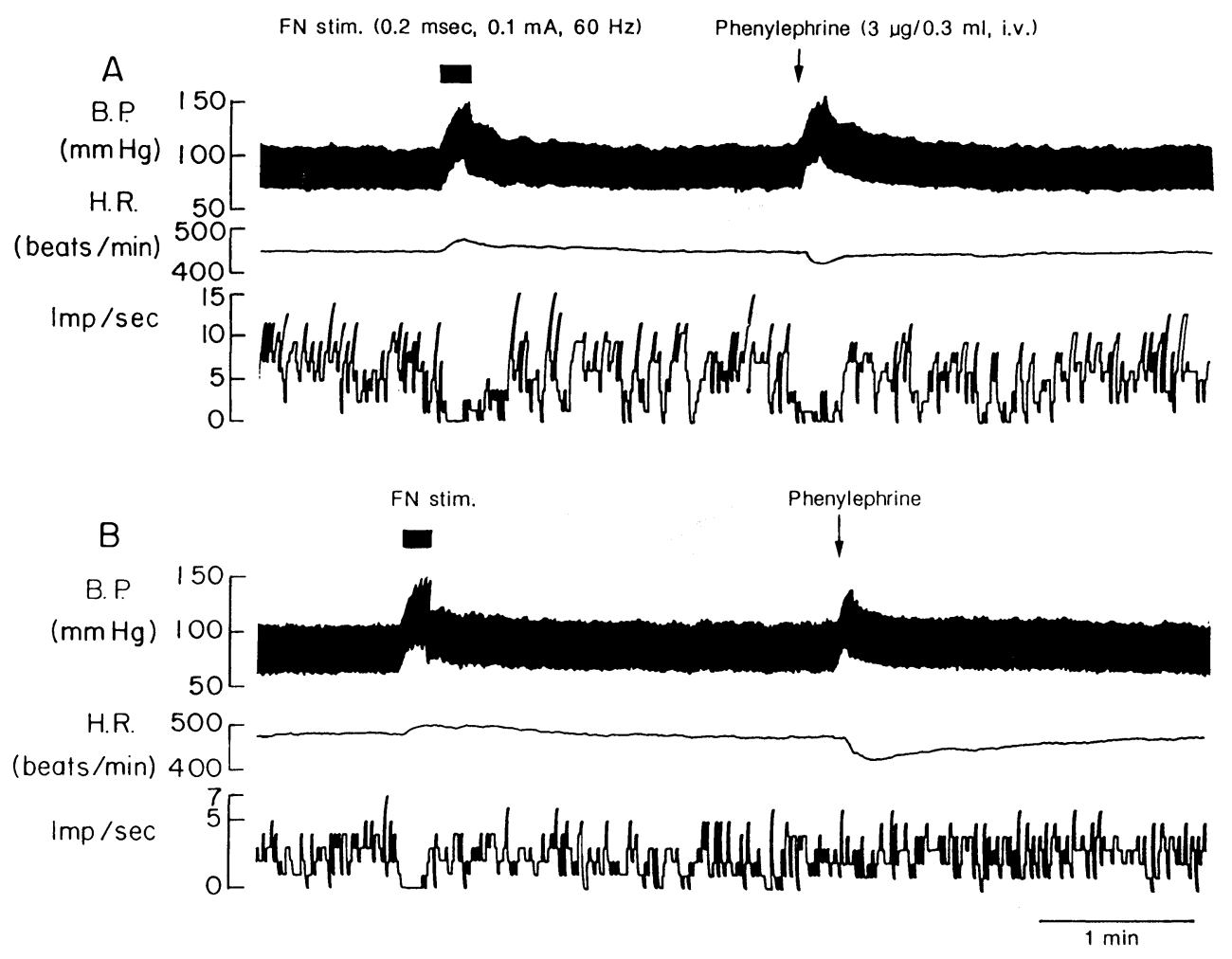

Fig. 2. The effects of train stimulation of the fastigial nucleus (FN) and an intravenous injection of phenylephrine on paraventricular neurosecretory cells. A and B, polygraph tracings showing the blood pressure (BP), heart rate (HR) and neuronal activity (number of spikes per second, Impulses/s). A: the neuronal activity was inhibited by FN stimulation (indicated by a filled rectangle, $0.2 \mathrm{~ms}$ duration, $0.1 \mathrm{~mA}$ intensity, $60 \mathrm{~Hz}$ for $10 \mathrm{sec}$ ), which produced a fastigial pressor response, and by an intravenous infusion of phenylephrine (the arrow, $3 \mu \mathrm{g}$ in $0.3 \mathrm{ml}$ Ringer solution), which produced hypertension and bradycardia. B: the neuronal activity was inhibited by FN stimulation but was not affected by phenylephrine infusion. From ref [8] with permission.

\section{Monosynaptic inhibition of neurosecretory cells by FN stimulation}

To further examine the synaptic nature of the fastigial inputs to the PVN, an in vivo intracellular recording from PVN neurons was performed in anesthetized rats [8]. Before the FN was stimulated, the membrane properties of the neurosecretory cells in the PVN were investigated and it was found that the input resistance of the neurosecretory cells was much higher than that of non-neurosecretory cells (117 M $\Omega$ vs. $53 \mathrm{M} \Omega$ ).

In response to $\mathrm{FN}$ stimulation, about half of the neurosecretory neurons exhibited postsynaptic potentials, of which more than $80 \%$ were inhibitory (IPSPs), and thus these findings were comparable with the results of extracellular recordings. Furthermore, about $80 \%$ of the IPSPs were considered to be monosynaptically evoked since their latencies remained constant when the FN stimulus intensity was changed (Fig. 3A). The reversal membrane potential of the IPSPs was approximately $-85 \mathrm{mV}$. Non-neurosecretory cells also receive inhibitory synaptic inputs from the FN, although they are polysynaptic.

Anatomical studies did not demonstrate any connections from the cerebellum to the PVN [6], but our results suggest the existence of direct pathways from the FN to the PVN. The exact route involved in the fastigio-hypothalmic connections is still unknown. We have shown that the lateral hypothalamic neurons also receive inhibitory monosynaptic projections from the FN. The inhib- 

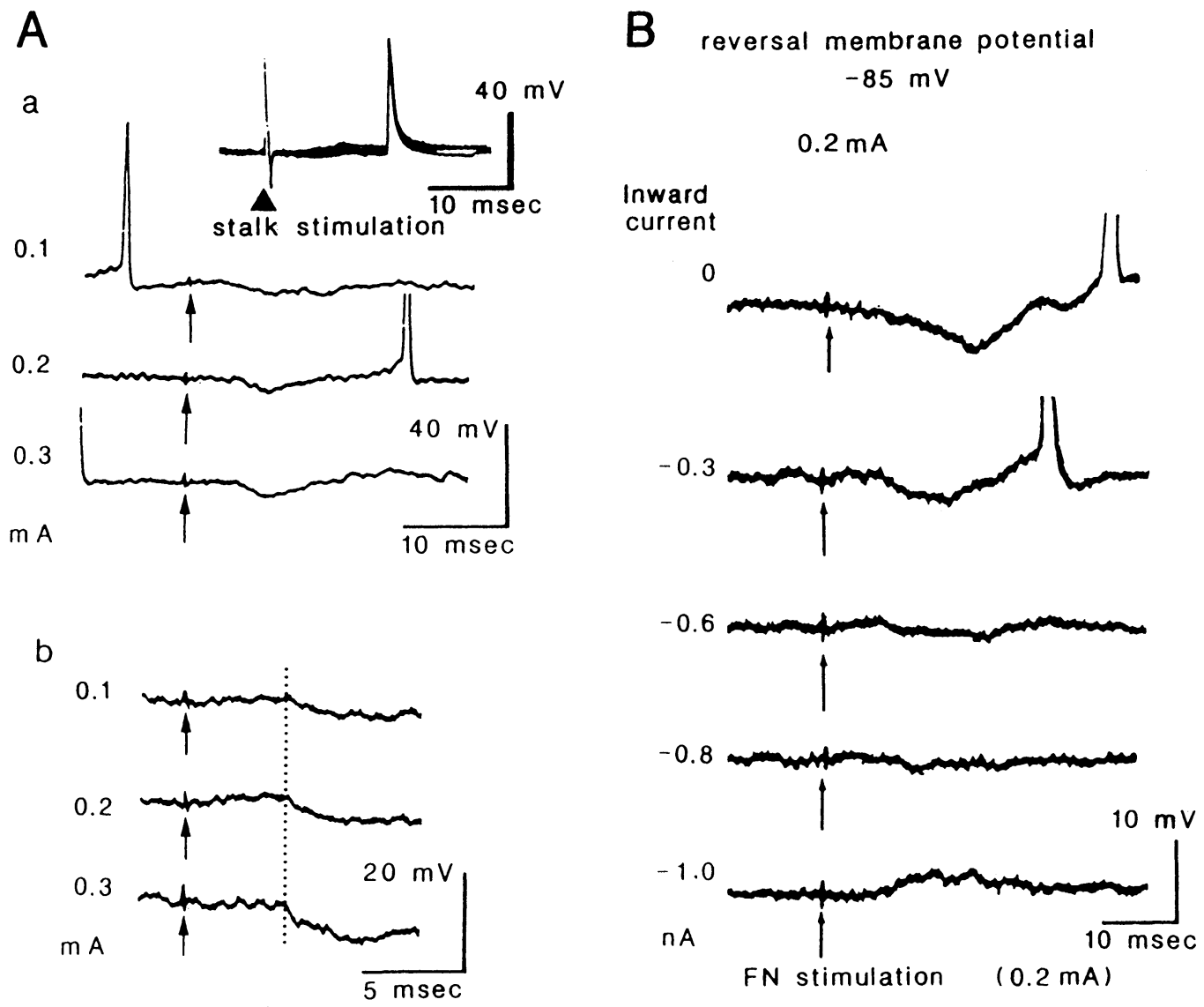

Fig. 3. Monosynaptic IPSPs evoked by stimulation of the fastigial nucleus (FN). A and B, from different neurons. The arrows indicate the time of FN stimulation $(0.2 \mathrm{msec}$ duration at $1 \mathrm{~Hz})$. Aa, inset: five superimposed tracings of antidromic action potentials following pituitary stalk stimulation (closed triangle). The IPSP amplitude was graded by increasing the stimulus intensity. Ab, latency of IPSP was constant when the stimulus intensity was increased. B: the application of the hyperpolarizing current resulted in decreases in the IPSP amplitude and a polarity change. Reversal potential was $-85 \mathrm{mV}$. From ref [8] with permission.

itory responses were unaffected by transection of the inferior cerebellar peduncle, although the pressor response was substantially abolished [7]. The fact that there are no efferent projections from the FN into the middle cerebellar peduncle suggests that fastigial projections to the hypothalamus may at least pass through the superior cerebellar peduncle.

\section{The Physiological Significance of the Cerebellar Input to the Hypothalamus}

The existence of monosynaptic inhibitory input from the FN to neurosecretory cells in the PVN is likely to have some physiological significance.
Stimulation of the FN evokes a defense reaction [34] in which the baroreflex may be strongly suppressed since both the blood pressure and heart rate rose during FN stimulation. In fact it has been shown that FN stimulation does inhibit the baroreflex [35]. Circulating vasopressin inhibits the sympathetic outflow [36] but accelerates the efferent vagal tone [37]. It is therefore reasonable to assume that in reactions, such as the defense or fastigial pressor response, neurally mediated inhibition of the baroreflex is also accompanied by inhibition of the vasopressin release by the hypothalamo-neurohypophyseal system. Another possibility for the role of the inhibitory influence of the FN on vasopressin release is to inhibit an excess secretion of vasopressin in an upright posi- 
tion [14].

It has been shown that the dorsal vagal complex (DVC) in the brain stem is innervated by vasopressinergic and oxytocinergic fibers, which, at least in part, originate in the PVN [38]. Since vagal motoneurons in the DVC are activated by the application of vasopressin through its direct action on the receptors [39], vasopressin released from nerve terminals is thought to enhance the vagal tone by working as neurotransmitter/neuromodulators. It has also been proposed that magnocellular neurons projecting to the posterior pituitary and parvocellular vasopressinergic neurons to the DVC could be co-activated by certain stimuli, such as hypovolemia, cellular dehydration and nausea [40, 41], suggesting co-operative actions of vasopressin in both the blood and brain. If non-neurosecretory cells recorded in our study contain the vasopressinergic neurons projecting to the brain stem, vasopressin released in the DVC would be reduced during FN stimulation, since non-neurosecretory cells also receive inhibitory input from the FN. This hypothesis may be consistent with the inhibitory effects of FN stimulation on baroreflex by reducing both the circulating and brain vasopressin concentrations.
Stimulation of the rostral portion of the FN has been found to evoke not only cardiovascular responses but also such complex behavior as grooming, feeding and attacking [42]. In addition to the direct connections between the FN and the PVN, anatomical and electrophysiological studies have shown that the FN is connected to several other parts of the hypothalamus such as the lateral hypothalamic area and the ventromedial nucleus $[6,7]$, which are known to play an important role in feeding and other emotional behavior. It is possible that the pathways between the cerebellum and the hypothalamus may therefore be important in modulating a number of different types of behavior.

\section{Summary}

The hypothalamo-neurohypophyseal system as well as the autonomic nervous system is involved in homeostatic responses associated with changes in head position and orthostatic reflex. The responses induced by body tilt on earth are thought to be attributed to changes in inputs from baroreceptors, vestibular organs and proprioreceptors that

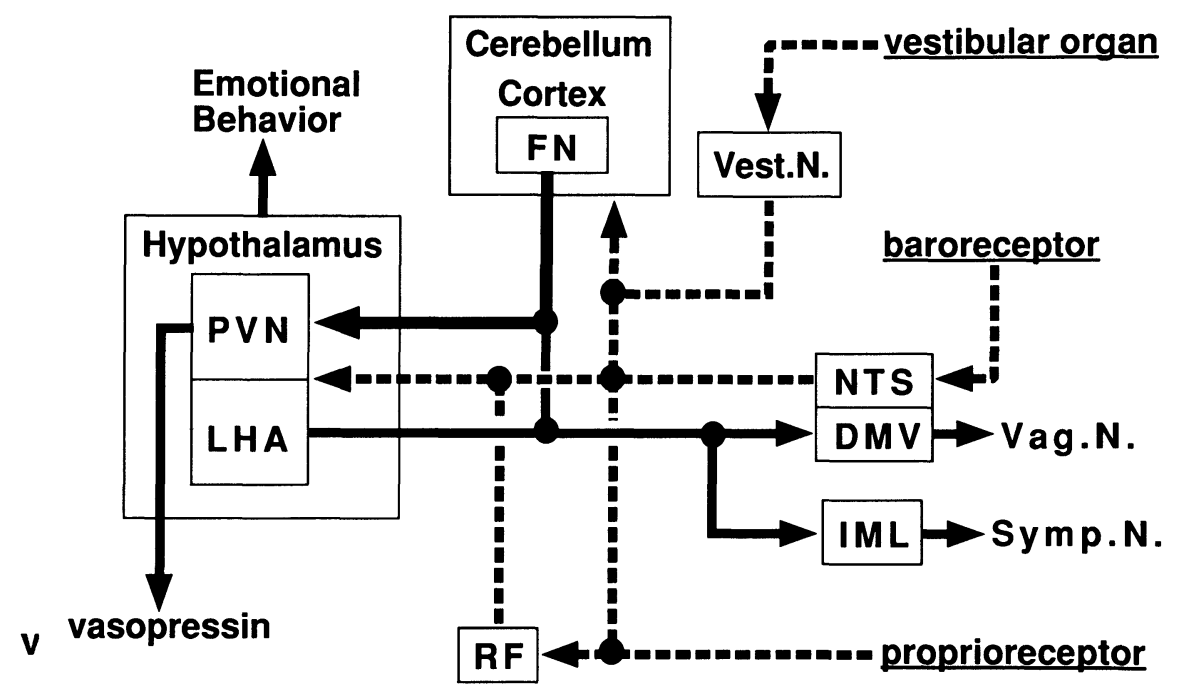

Fig. 4. Input-output organization between the hypothalamus, cerebellum and brain stem. Afferents (dotted lines) to and efferents (solid lines) from the hypothalamus and cerebellum. LHA, lateral hypothalamic area; PVN, paraventricular nucleus; FN, fastigial nucleus; Vest. N., vestibular nucleus; NTS, nucleus tractus solitarius; DMV, dorsomotor nucleus of the vagus; IML, intermediolateral cell column of the spinal cord; RF, reticular formation; Vag. N., vagus nerve; Symp. N., sympathetic nerve. 
are normally required for postural control. The information from these organs is sent to the hypothalamus which thereby influences both neuroendocrine and autonomic systems as well as various kinds of emotional behavior. Our findings showing the fastigial input to the hypothalamus suggested that the FN plays a significant role in these homeostatic responses through its connections with the brain stem and the hypothalamus. Figure 4 shows the input-output organization among the hypothalamus, cerebellum and brain stem, described in detail in sections III to V. This hypothesis may help to account for the autonomic and endocrine disorders often observed in weightlessness.

\section{Acknowledgement}

We thank Dr. B.T. Quinn, Kyushu University, for his help in preparing the manuscript.

\section{References}

1. Palkovits J (1986) Afferents onto neuroendocrine cells. In: Ganten D, Pfaff D (eds) Morphology of hypothalamus and its connections. Current Topics in Neuroendocrinology. Springer-Verlag, Berlin, vol 7: 197-222.

2. Kannan H, Yamashita H (1983) Electrophysiological study of paraventricular nucleus neurons projecting to the dorsomedial medulla and their response to baroreceptors stimulation in rats. Brain Res 279: 31-40.

3. Koizumi K, Yamashita H (1978) Influence of atrial stretch receptors on hypothalamic neurosecretory neurones. J Physiol (Lond) 285: 341-358.

4. Brooks CM, Ushiyam J, Lange GR (1962) Reactions of neurons in or near the supraoptic nuclei. $A m J$ Physiol 202: 487-490.

5. Feldman S (1963) Effects of reticular formation lesions on afferent projections to the hypothalamus. Electroencephologr Neurophysiol 15: 672-682.

6. Haines DE, Dietrichs E (1987) On the organization of interconnections between the cerebellum and hypothalamus. In: King JS (ed) New Concepts in Cerebellar Neurobiology. Alan R Liss, New York, 113-149.

7. Min B-I, Oomura Y, Katafuchi T (1989) Response of rat lateral hypothalamic neuronal activity to fastigial nucleus stimulation. J Neurophysiol 61: 1178-1184.

8. Katafuchi T, Koizumi K (1990) Fastigial inputs to paraventricular neurosecretory neurones studied by extra- and intracellular recordings in rats. J Physiol (Lond) 421: 535-551.

9. Sandler H (1979) Low-G simulation in mammalian research. Physiologist 22: S19-S22.

10. Katafuchi T, Oomura Y, Aoyagi K (1987) Single neuron activity of rat hypothalamic paraventricular nucleus during body suspension. Neurosci Lett 78: 301-306.

11. Nixon JV, Murray RG, Bryant C, Johnson RL, Mitchell JG, Holland OB, Gomez-Sanchez C,
Vergne-Marini P, Blomqvist CG (1979) Early cardiovascular adaptation to simulated zero gravity. J Appl Physiol 46: 541-548.

12. Convertino VA, Benjamin BA, Keil LC, Sandler H (1984) Role of cardiac volume receptors in the control of ADH release during acute simulated weightlessness in man. Physiologist 27: S51-S52.

13. Ghelarducci B (1973) Responses of the cerebellar fastigial neurones to tilt. Pflügers Arch 344: 195206.

14. Hata N, Miura M (1974) The inhibitory effect of the cerebellar fastigial stimulation on $\mathrm{ADH}$ secretion. J Physiol (Lond) 242: 793-803.

15. Del Bo A, Sved AF, Reis DJ (1983) Fastigial stimulation releases vasopressin in amounts that elevate arterial pressure. Am J Physiol 244: H687-H694.

16. Katafuchi T, Yoshimatsu Y, Oomura Y (1984) Responses of lateral hypothalamic neurons to simulative hypogravic condition induced by body suspension. Brain Res Bull 12: 29-31.

17. Kannan $\mathrm{H}$, Yamashita H, Koizumi K, Brooks CM (1988) Neuronal activity of the cat supraoptic nucleus is influenced by muscle small-diameter afferent (groups III and IV) receptors. Poc Natl Acad Sci USA 85: 5744-5748.

18. Ricardo JA, Koh ET (1978) Anatomical evidence of direct projections from the nucleus of the solitary tract to the hypothalamus, amygdala, and other forebrain structures in the rat. Brain Res 153: 1-26.

19. Koh ET, Ricardo JA (1975) Afferents and efferents of the parabrachial region in the rat: evidence for parallel ascending gustatory versus visceroceptive systems arising from the nucleus of the solitary tract. Anat Rec 190: 449.

20. Wolf G, DiCara, LV (1971) A third ascending hypothalamopetal pathway. Exp Neurol 33: 69-77.

21. Katafuchi T, Puthuraya KP, Yoshimatsu H, Oomura Y (1987) Responses of rat lateral hypothalamic neuron activity to vestibular nuclei stimulation. Brain 
Res 400: 62-69.

22. Somana R, Walberg F (1979) Cerebellar afferents from the nucleus of the solitary tract. Neurosci Lett 11: 41-47.

23. Moolenaar GM, Rucker HK (1976) Autoradiographic study of brain stem projections from fastigial pressor areas. Brain Res 114: 492-496.

24. Batton RR, Jayaraman A, Ruggiero D, Carpenter MB (1977) Fastigial efferent projections in the monkey: an autoradiographic study. J Com Neurol 174: 281-306.

25. Ross CA, Ruggiero DA, Reis DJ (1981) Afferent projections to the cardiovascular portions of the nucleus of the tractus solitarius in the rat. Brain Res 223: 402-408.

26. Dietrichs E (1984) Cerebellar autonomic function: direct hypothalamocerebellar pathway. Science 223: 591-593.

27. Miura M, Reis DJ (1969) Cerebellum: a pressor response elicited from the fastigial nucleus and efferent pathways in brain stem. Brain Res 13: 595599.

28. Nakai M, Iadecola C, Reis DJ (1982) Global cerebral vasodilation by stimulation of rat fastigial cerebellar nucleus. Am J Physiol 243: H226-H235.

29. Doba N, Reis D (1972) Changes in regional blood flow and cardiodynamics evoked by electrical stimulation of the fastigial nucleus in the cat and their similarity to orthostatic reflexes. J Physiol (Lond) 227: 729-747.

30. Miura M, Takayama K (1988) The site of origin of the so-called fastigial pressor response. Brain Res 473: 352-358.

31. Henry RT, Connor JD (1989) Axons of passage may be responsible for fastigial nucleus pressor response. Am J Physiol 257: R1436-R1440.

32. Dormer K, Foreman R, Stone H (1977) Glutamateinduced fastigial pressor response in the dog. Neuroscience 2: 577-584.
33. Sved AF, McdDowell FH, Blessing WW (1985) Release of antidiuretic hormone in quadriplegic subjects in response to head-up tilt. Neurology 35: 78-82.

34. Zanchetti A, Zoccolini A (1954) Autonomic hypothalamic outbursts elicited by cerebellar stimulation. J Neurophysiol 17: 475-483.

35. Lisander B, Martner J (1971) Interaction between the fastigial pressor response and the baroreceptor reflex. Acta physiol scand 83: 505-514.

36. Nishida Y, Bishop VS (1992) Vasopressin-induced suppression of renal sympathetic outflow depends on the number of baroafferent inputs in rabbits. Am J Physiol 263: R1187-R1194.

37. Curtice GP, Kwong TE, Lumbers ER, Potter EK (1984) Excitation of the cardiac vagus by vasopressin in mammals. J Physiol (Lond) 354: 547-556.

38. Sofroniew MV, Schrell U (1981) Evidence for a direct projection from oxytocin and vasopressin neurons in the hypothalamic paraventricular nucleus to the medulla oblongata: immunohistochemical visualization of both the horseradish peroxidase transported and the peptide produced by the same neurones. Neurosci Lett 22:211-217.

39. Mo Z-I, Katafuchi T, Muratani H, Hori T (1992) Effects of vasopressin and angiotensin II on neurones in the rat dorsal motor nucleus of the vagus, in vitro. I Physiol (Lond) 458: 561-577.

40. Riphagen CL, Pittman OJ (1986) Arginine vasopressin as a central neurotransmitter. Fed Proc 45: 2318-2322.

41. McCann MJ, Rogers RC (1990) Oxytocin excites gastric-related neurones in rat dorsal vagal complex. J Physiol (Lond) 428: 95-108.

42. Reis DM, Doba N, Nathan MA (1973) Predatory attack, grooming, and consummatory behaviors evoked by electrical stimulation of cat cerebellar nuclei. Science 182: 845-847. 